\title{
La promesa de felicidad en los mensajes de la publicidad comercial
}

\author{
María Teresa Pellicer JoRdÁ ${ }^{1}$ \\ Universidad de Murcia
}

Recibido: 03/01/2013

Aceptado: 25/05/2013

\begin{abstract}
Resumen
La publicidad ha tenido que adaptarse, desde su nacimiento, a los cambios sociales y eso le obliga a cambiar de forma constante las ideas con las que llamar la atención del consumidor. En este artículo vamos a estudiar una de las ideas primordiales en la filosofía publicitaria actual: la felicidad publicitaria. Este es el concepto que se utiliza en la mayoría de anuncios -bien sea de forma implícita o explícita- que podemos ver en la actualidad. Vamos a estudiar en qué consiste este concepto, cuándo nace, cómo se ha configurado, así como sus formas de implantación en la publicidad actual.
\end{abstract}

Palabras Clave: Publicidad; felicidad; filosofía; ética; sociedad.

\section{The promise of happiness in the messages of commercial publicity}

\begin{abstract}
From the beginning publicity has had to adapt itself to social changes. As a consequence, it has been constantly forced to change its ideas in order to call the attention of consumers. In this article we will study one of the main ideas of the philosophy of publicity as we know it nowadays: happiness in publicity. This concept is used either in an implicit or an explicit way in most of current advertisements. Thus, we will study the meaning of this concept, its origins and the way it was shaped, together with the different ways in which it is being introduced in current publicity.

Key Words: Advertising; happiness; philosophy; ethics; society.

${ }^{1}$ María Teresa Pellicer Jordá es Doctora por la Facultad de Comunicación y Documentación de la Universidad de Murcia, centro en el que obtuvo las licenciaturas de Periodismo y Publicidad y Relaciones Públicas. Ha escrito dos libros, titulados 'Ética y estrategias publicitarias' y 'La publicidad como industria cultural'. Actualmente trabaja como profesora en la Facultad de Comunicación y Documentación de la Universidad de Murcia.
\end{abstract}




\section{Origen de la felicidad publicitaria}

La publicidad cuenta con una larga historia que atestigua su creciente importancia en la sociedad. Existen varias teorías acerca de cuándo aparece la publicidad. Como explica Checa Godoy $^{2}$, hay autores que sitúan sus inicios en la antigüedad, mientras que otros consideran que no podemos hablar de publicidad hasta la Revolución Industrial, etapa que le otorga un carácter masivo. En cualquier caso, a lo largo de esa historia -más o menos larga según el autor-, ha ido perfilando procedimientos para conseguir su objetivo básico y fundamental- razón de su existencia por otro lado- que no es otro que fomentar las ventas. Como dice Ortega ${ }^{3},<<$ el mensaje publicitario representa el conjunto de ideas que el anunciante desea hacer llegar a un público previamente definido para conseguir los objetivos establecidos $>>$. Esas ideas son las que se van a estudiar en este artículo y dentro de ellas, se va a analizar la idea fundamental y básica sobre la que gira la filosofía publicitaria actual, presente en casi todos los anuncios que podemos ver actualmente: la felicidad. Ese concepto o gran valor va a ir apareciendo en la publicidad conforme ésta se va desarrollando, hasta conseguir una presencia tan relevante, que se convierte, en la mayoría de casos, en el argumento principal de venta.

La primera pregunta que se debe plantear es si este concepto supremo de felicidad como centro de la actividad publicitaria ha estado siempre presente o ha aparecido en un momento determinado. En Egipto, donde sitúan el primer anuncio conocido, no se encuentra ninguna referencia a la felicidad publicitaria, como explica Godoy ${ }^{4}$. Lo mismo ocurre en el caso de los fenicios o de los griegos, aunque en este último caso ya aparece el elemento simbólico en la publicidad que realizaban. En Roma continúan con esta tónica general, ya que la publicidad fomenta las ventas únicamente anunciando un producto y definiendo con cierta claridad las características de los mismos, con cierto carácter simbólico, pero sin aludir al concepto que se estudia en este artículo. Como apunta Eguizábal ${ }^{5},<<$ el principal argumento de venta sigue siendo la mercancía misma. A finales de la Edad Media, se inicia el alejamiento de la materialidad del producto mediante representaciones icónicas de los mismos $>>$.

El momento en el que aparece la imprenta constituye un antes y un después en la actividad publicitaria, ya que permite otorgarle un carácter masivo. A raíz de ahí, aparecen los periódicos y otros medios de comunicación, que se convierten en los aliados fundamentales de la publicidad. Le sigue la aparición de las marcas. Poco a poco va aumentando la actividad publicitaria e incluso se pueden ver los primeros anuncios en los que lo que se promete no tiene mucho que ver con la realidad. Con la aparición de la televisión, $<<$ la publicidad empieza a introducir el color como elemento principal (componente importante del concepto de felicidad) $>>$. Analizando anuncios de estas épocas, se puede concluir que la aparición de la felicidad publicitaria se encuentra en el momento en el que la publicidad adquiere un carácter masivo, ya que es entonces cuando se empiezan a ver anuncios con personas sonrientes, felices por consumir ese producto o servicio que se ofrece. Es, eso sí, una felicidad sugerida de forma implícita,que más tarde se verá reforzada con la aparición de la televisión y del

${ }^{2}$ CHECA GODOY, A. (2007): Historia de la publicidad, Galicia, Netbiblo, 3.

${ }^{3}$ ORTEGA, E. (2004): La comunicación publicitaria, Madrid, Pirámide, 217.

${ }^{4}$ CHECA GODOY, A. (2007): Historia de la publicidad, Galicia, Netbiblo, 3.

${ }^{5}$ Ibidem, 48. 
color. Hellín ${ }^{6}$ explica que $<<$ poco a poco, la publicidad empieza a convertirse en lo que hoy conocemos, mucho más reflexiva y racional de lo que era en sus comienzos, dando lugar a una publicidad que permite diferenciar a las personas $>>$, a través de los productos que ellas consumen, como afirma Roberts ${ }^{7}$, y la felicidad se convierte en el elemento diferenciador principal. Eguizábal ${ }^{8}$ ratifica esta idea cuando dice que $<<$ con el paso de los años, la publicidad fue abandonando sus planteamientos raciones, tan decimonónicos por otro lado, para ofrecer otro tipo de beneficios de carácter más emocional $>>$. Ese carácter emocional es que el marca y define la publicidad actual, siendo la felicidad su baluarte más preciado y base de sus conquistas. La publicidad ha dejado ya de hablar de mercancías, para hablar de las personas, como afirma Eguizábal ${ }^{9}$. Es entonces cuando $<<$ la imagen se convierte también en más importante que el sujeto $>>$.

\section{Cómo creamos el concepto de felicidad publicitaria}

Una vez fijado el inicio de la felicidad publicitaria, la segunda cuestión que se debe plantear es cómo se consigue crear ese concepto. Cuando aparece este concepto como tal, lo hace de una forma implícita en los anuncios, para después hacerlo de una forma muy explícita, como se podrá ver a lo largo de este artículo. En cualquier caso -explica Eguizábal ${ }^{10}$ - el proceso de creación de esa felicidad publicitaria sería el siguiente:

Para que actúe el dispositivo publicitario hay que partir, pues, de un cierto grado de insatisfacción en los individuos. Su primer objetivo es por tanto producir en el consumidor un cierto disgusto, una desazón para con su vida, sus propiedades, su físico o sus relaciones. (...) Una vez creada esa conciencia, la solución se presenta fácil, porque ahí está siempre el mensaje que viene a aliviarnos, a consolarnos, a proporcionarnos el medio para librarnos de tal problema. La publicidad se preocupa por ti, está para ayudarte en tus carencias, para orientarte en tus conductas, para señalarte lo correcto y lo incorrecto, para que, en fin, no necesites pensar demasiado. Ahora bien, ¿de qué manera habla la publicidad de los consumidores? (...) Los anuncios definen las formas de la felicidad, nos señalan lo que debemos desear, aquello que compone el paraíso digno de ser recobrado. La meta no es el coche, sino las parejas que podemos conquistar con él, no es el perfume sino las parejas que lograremos seducir, no es el limpiador sino el asombro de nuestra suegra. La meta no es el producto, sino el anuncio.

Siguiendo a Eguizábal o a autores como Haas o Moles, según Hellín ${ }^{11}$, es necesario, por tanto, crear primero una insatisfacción, ya que como dice M. Scott Peck ${ }^{12}$ :

${ }^{6}$ HELlíN, P. A. (2006): Publicidad y valores posmodernos, Madrid, Miranda Comunicación, 64.

${ }^{7}$ ROBERTS, K. (2005): Lovemarks, el futuro más allá de las marcas, Barcelona, Urano, 25-30.

${ }^{8}$ EGUIZÁBAL, R. (2009): Industrias de la conciencia: una historia social de la publicidad en España (1975-2009), Barcelona, Península, 20.

${ }^{9}$ Ibídem, 17.

${ }^{10}$ EGUIZÁBAL, R (2009): Industrias de la conciencia: una historia social de la publicidad en España (1975-2009), Barcelona, Península, 21.

${ }^{10}$ HELLÍN, P. A (2006): Publicidad y valores posmodernos, Madrid, Miranda Comunicación, 132 y 133 .

${ }^{11}$ ROVIRA, A. Y MIRALLES, F. (2008): Ideas sobre la felicidad, Madrid, Punto de Lectura, 169. 
Nuestros momentos de más lucidez suelen tener lugar cuando nos sentimos profundamente incómodos, infelices o insatisfechos. Pues es en estos momentos empujados por nuestra insatisfacción cuando salimos del camino trillado y empezamos a explorar maneras diferentes de hacer algo o respuestas más certeras.

Ahí, en ese momento de exploración de nuevas formas, en esa búsqueda de lo que falta al ciudadano, es donde actúa la publicidad, poniendo toda su artillería pesada. Ahora bien, si se crea la insatisfacción o ya se tiene creada, ¿cómo se ofrece la solución al consumidor?, ¿cómo se le hace llegar en medio de la fuerte competencia que existe? Es fácil, como explica Miñana ${ }^{13}$, cuando dice que:

Hay que emocionar al consumidor, aunque no se deje, entrar como sea en la lista corta de sus afectos. Hay que saltar más alto, gritar más fuerte, impresionar a cualquier precio su retina. Las marcas poderosas optan por la omnipresencia para que no se les suba a las barbas algunas de las marcas registradas.

Todas las marcas, todos los productos y servicios ofrecen la felicidad como ventaja de compra principal, ya sea de forma implícita o de forma explícita, de manera que emocionar, llegar hasta el corazón del consumidor, se convierte en la única manera de destacar dentro de la gran competencia existente, dentro de un mercado con productos tan similares, que no tiene otra opción que recurrir al universo simbólico de la publicidad, a valores y emociones, para poder distinguirse del resto. Es como apunta Magariños de Morentín ${ }^{14}$ cuando dice que:

Estas representaciones mentales colectivas -que crea la publicidad- que por su fuerte carga emotiva canalizan o hacen converger aspiraciones y deseos, activan miedos o temores en varios sectores de la población, promueven o inhiben comportamientos, contribuyendo por eso a veces a la creación de modernas mitologías.

Esto es así porque el hombre tiene la necesidad de ser feliz. Como explica Zubiri ${ }^{15}$ $<<$ las ilusiones, las desilusiones, los proyectos fallidos de que está compuesta y tejida la vida, hacían decir a Bergson que el camino de nuestra vida está bordeado por las ruinas de lo que pudimos haber sido y no fuimos $>>$. La publicidad se ha convertido, de esta forma, en la garantía que permite a la persona ser lo que no fue, la que permite ser lo que se desea ser y, además, de una forma muy sencilla, como se verá a lo largo del artículo.

\section{3. ¿Qué es la felicidad publicitaria?}

La siguiente cuestión es por qué la publicidad ha elegido a la felicidad como su argumento principal de venta, ya que existen otros muchos elementos inmateriales a los que poder hacer referencia y con los que poder vender los productos y servicios que hay actualmente en el mercado. La respuesta a esta cuestión es fácil: la felicidad es lo que todo ser humano ansía. Todo se puede reducir a si uno es feliz o no, porque esa felicidad incluye el resto de cosas de la vida.

${ }^{13}$ MIÑANA, J (2003): El mono cansado. Reflexiones sobre publicidad, Barcelona, Sine Qua Non, 49.

${ }^{14}$ MADRID CÁNOVAS, S. (2006): Semiótica del discurso publicitario, Murcia, Universidad de Murcia, 108.

${ }^{15}$ ZUBIRI, X. (2005): El hombre, lo real y lo irreal, Madrid, Alianza Editorial, 10. 
Esa felicidad, como objetivo y necesidad común del ser humano, se puede encontrar en todas las corrientes éticas y filosóficas de la historia. Haciendo un breve repaso por ellas se verá cómo se proponen maneras, a veces muy distintas, para alcanzarla. Para Sócrates ${ }^{16}$, la única forma de alcanzar la felicidad era a través de la moral, mientras que para Platón ${ }^{17}$, la felicidad es posible cuando el hombre es capaz de llegar hasta las ideas, es decir, hasta la esencia de las cosas. Constituyen estos dos conceptos muy profundos de lo que constituye la felicidad, contrarios como se verá, a esa felicidad tan externa que ofrece la publicidad. Aristóteles ${ }^{18}$ comparte esa concepción profunda y en sus planteamientos afirma que la felicidad -como el mayor bien que puede tener el hombre- era $<<$ la plenitud de la realización activa del hombre $>>$, como explica Marías ${ }^{19}$, y se alcanza a través de la virtud. Se puede observar como $<<$ entre los filósofos de la antigua Grecia, lo moral se concibe como la búsqueda de la felicidad o la vida buena $>>$, como explica Cortina ${ }^{20}$.

Los epicúreos ofrecen una nueva visión de la felicidad, en la que lo material cobra protagonismo. ¿Cómo conseguirla? Consideran que se consigue buscando el placer, ya sea material o inmaterial, y evitando el dolor, como afirma Marías ${ }^{21}$. Visión parecida es la que ofrecen los utilitaristas, como Mill22, que proponen el concepto de Mayor felicidad, que dice que las acciones son correctas en la medida en que tienden a promover la felicidad, entendida ésta como el $<<$ placer o ausencia de dolor $>>$, tal y como explica Robinson-Chriss ${ }^{23}$, aunque tanto en el caso de los epicuros como en el de los utilitaristas, esos placeres tienen que cumplir una serie de requisitos muy estrictos, que los alejan de la superficialidad.

Se observa como la búsqueda de la felicidad es, y siempre ha sido, protagonista del pensamiento del hombre y de la filosofía, que la ha considerado como algo positivo y razonable para el ser humano. Aunque $\mathrm{Kant}^{24}$ le quita importancia, diciendo que $<<$ cuanto más se preocupa una razón cultivada del propósito de gozar la vida y alcanzar la felicidad, tanto más el hombre se aleja de la verdadera satisfacción $>>$. En cualquier caso y, aunque le reste importancia en relación a otras corrientes, Kant ${ }^{25}$ sí propone cómo alcanzar la felicidad y afirma que:

Nadie es capaz de determinar, por un principio, con plena certeza que sea lo que le haría verdaderamente feliz. (...) Así pues para ser feliz no cabe obrar por principios determinados, sino sólo por consejos empíricos: por ejemplo: la dieta, de ahorro, de cortesía, de comedimiento, etc. La experiencia enseña que estos consejos son los que mejor fomentan, por término medio, el bienestar.

\footnotetext{
${ }^{16}$ JENOFONTE. (1967). Recuerdos de Sócrates, Madrid, Alianza Editorial, 169.

${ }^{17}$ PLATÓN, (1989): La República, Barcelona, Edit. Ceac.

${ }^{18}$ ARISTÓTELES, (2000): Metafísica, Madrid, Biblioteca Básica Gredos.

${ }^{19}$ MARÍAS, J. (1941): Historia de la filosofía, Madrid, Alianza Editorial, 103.

${ }^{20}$ CORTINA, A. Y MARTÍNEZ, E. (2008): Ética, Madrid, Akal, 31.

${ }^{21}$ MARÍAS, J. (1941): Historia de la filosofia, Madrid, Alianza Editorial, 115

${ }^{22}$ MILL, J.S. (2007): El utilitarismo, Madrid, Alianza Editorial, 49-50.

${ }^{23}$ ROBINSON- CHRISS, D. (2005): Ética para todos,. Barcelona, Paidós, 71.

${ }^{24}$ KANT, I. (1967): Fundamentación de la metafísica de las costumbres, Madrid, Colección Austral, 30.

${ }^{25}$ Ibidem. Pág. 67
} 
La felicidad es, por tanto, uno de los aspectos más importantes de la vida humana. Citando a Adela Cortina ${ }^{26}$, se puede resumir diciendo que:

Sin embargo, ya entre los griegos hubo discrepancias sobre el modo de interpretar la felicidad (...) En los tiempos modernos todavía persiste la corriente hedonista, representada especialmente por el utilitarismo. En efecto, muchos filósofos utilitaristas continúan identificando la felicidad por el placer, y el placer a su vez es entendido como sensación agradable, como satisfacción sensible. Frente a ellos, otras escuelas contemporáneas insisten en afirmar que, si bien la felicidad es algo a lo que todos los hombres aspiran, no es cierto que esa aspiración consista en experimentar sensaciones placenteras. Sin renunciar necesariamente el placer, lo que en realidad mueve a los seres humanos es la búsqueda de felicidad entendida como autorrealización

Al margen de las discrepancias, la felicidad sigue siendo el eje central del hombre, lo que hace afirmar a Powell ${ }^{27}$ que $<<$ la condición natural de los seres humanos es la felicidad. (...) Yo creo que todos sentimos un innato y persistente deseo de ser felices $>>$.

Esta necesidad innata de felicidad que todos los seres humanos tienen es una de las razones por las que la publicidad ha convertido a ésta en uno de sus argumentos de venta principales. De hecho, se puede hablar de una filosofía publicitaria respecto a la felicidad, que podría ser la siguiente: para ser feliz, lo único necesario es confiar en uno mismo, en los anuncios y comprar sus productos. Aunque lo cierto es que esta es una felicidad pasajera, con fuente en el exterior del hombre y muy alejada a de las recetas de felicidad que, según se ha visto, plasman los distintos autores de la antigüedad o los actuales estudiosos de ella. De hecho, W. Beran Wolfe, según Rovira y Miralles ${ }^{28}$, decía que:

Si observas una persona realmente feliz, la encontrarás construyendo un barco, escribiendo una sinfonía, educando a sus hijos, plantando dalias en su jardín o buscando huevos de dinosaurio en el desierto de Gobi. No la encontrarás buscando la felicidad como si fuera la cuenta de un collar que se ha deslizado bajo el radiador.

Esa felicidad supone un concepto muy superficial -lo que ha venido a configurar la cultura light, según Powell ${ }^{29}$ - y efímero, definido este último por el Principito ${ }^{30}$ como algo que $<<$ está amenazado por una próxima desaparición $>>$. La felicidad publicitaria es así, acaba pronto, aunque si no se para de consumir sus productos, enlazaremos una felicidad con otra. Además, la publicidad parece haber tomado como suya la filosofía de vida que ya planteó en su tiempo Walt Disney ${ }^{31}$, cuando decía:

No creo que haya ninguna cumbre que no pueda ser escalada por una persona que conozca el secreto para hacer realidad sus sueños. Este secreto especial yo lo resumo en cuatro C. Son la curiosidad, la confianza, el coraje y la constancia. De todos, el más importante es la confianza. Cuando creas en algo, créelo sin dudar y cuestionarlo.

${ }^{26}$ KANT, I. (1967): Fundamentación de la metafísica de las costumbres, Madrid, Colección Austral, 30.

${ }^{27}$ POWELL, J. (1996): La felicidad es una tarea interior, Cantabria, Sal Terrae, 7.

${ }^{28}$ ROVIRA, A. Y MIRALLES, F. (2008): Ideas sobre la felicidad, Madrid, Punto de Lectura, 164.

${ }^{29}$ COSTA, J (1992): Reinventar la publicidad. Reflexiones desde las Ciencias Sociales, Madrid, Fundesco, 127-128.

${ }^{30}$ SAINT- EXUPÉRY, A. (1998): El principito, Madrid, Alianza Amecé, 68

${ }^{31}$ ROVIRA, A. Y MIRALLES, F. (2008): Ideas sobre la felicidad, Madrid, Punto de Lectura, 179. 
La publicidad tiene esas 4 ces: llama a la curiosidad del consumidor, le pide confianza en ella, le transmite la idea de coraje por la vida y es tan constante en el lanzamiento de su mensaje que al final acaba consiguiendo sus objetivos. Ahora bien, esa superficialidad de la que se habla al principio del párrafo es la que hace que la publicidad llegue a frustrar a los consumidores, como consecuencia de no ver éstos cumplidas sus expectativas, algo que nunca se debe hacer según Bassat ${ }^{32}$, que defiende que $<<$ la buena publicidad contiene una fuerte idea de venta y promete un beneficio interesante y alcanzable para el consumidor $>>$. En este sentido, Powell ${ }^{13}$ explica que:

Por supuesto, las expectativas tienen mucho que ver con nuestra felicidad y ésta es una de las lecciones vitales más difíciles de aprender. (...) Quizá nos haya ofuscado el maremoto de publicidad que nos inunda y nos asegura que seremos felices si compramos y usamos determinados productos: tendremos buen aspecto, daremos buena impresión, oleremos bien; en suma, conduciremos por las autopistas de la vida con una feliz e imprudente despreocupación. Estos reclamos publicitarios quieren hacernos creer que la felicidad no es más que una multiplicación de placeres. De modo que nos hemos endeudado consumiendo todos los productos portadores de felicidad.

Quizá es lo mismo que le ocurrió al ciudadano $\mathrm{Kane}^{34}$, que:

Se dedicó, durante muchos años a vender a todas las personas para poder comprarse todas las cosas; al final de su vida reconoce que cambiaría, si pudiera su almacén repleto de cosas carísimas por la única cosa humilde -un viejo trineo- que le recordaba a cierta persona: a él mismo, antes de dedicarse a la compraventa, cuando prefería amar y ser amado que poseer o dominar.

$\mathrm{Al}$ margen de esas frustraciones, esa felicidad publicitaria -como hemos considerado a bien llamarla- está totalmente instaurada en la sociedad. El libro Un mundo feliz, de Huxley ${ }^{35}$, supone una aproximación muy acertada de lo que está ocurriendo en la sociedad actual. De este modo, en una de sus páginas podemos leer: $<<$ Y he aquí- dijo el director sentenciosamente- el secreto de la felicidad y la virtud: amar lo que hay obligación de hacer. Tal es el fin de todo el condicionamiento: hacer que cada uno ame el destino social, del que no podrá librarse $>>$. La obligación de cualquier persona es, por tanto, consumir. Ésta es la base del capitalismo y una de las funciones primordiales del ciudadano.

\section{4. ¿Cómo se configura el concepto?}

La siguiente cuestión es ver cómo vende la publicidad ese concepto de felicidad y lo hace de dos formas principales:

- Hablando explícitamente del concepto de felicidad

- Hablando de forma implícita del concepto de felicidad, a través de la actitud de los personajes que aparecen en las escenas del anuncio, bien sea a través de gestos de felicidad o del concepto de éxito.

En la primera de las opciones (concepto explícito de felicidad), cada vez son más las marcas que se atreven a nombrar a la felicidad de una forma explícita en sus anuncios, convirtiéndose ellas en estandartes de esta codiciada emoción.

${ }^{32}$ BASSAT, L. (1998): El libro rojo de la publicidad, Madrid, Espasa, 15 y 85

${ }_{33}^{3}$ POWELL, J. (1996): La felicidad es una tarea interior, Cantabria, Sal Terrae, 7.

${ }^{34}$ SAVATER, F. (1998): Ética para Amador, Barcelona, Ariel, 85.

${ }^{35}$ HUXLEY. A. (1995): Un mundo feliz, México, Editores Mexicanos Unidos, 25. 
Uno de los casos más ejemplificadores es el de Coca Cola ${ }^{36}$, que se atrevió a mandar a embajadores de la felicidad por todo el mundo para descubrir qué es lo que hace feliz a la gente, lo que lleva a Eguizábal ${ }^{37}$ a decir que $<<$ no es Coca-Cola quien te hace feliz, es el anuncio de Coca-Cola >>. Esta marca es la que, quizá, haya explotado mejor y hecho suyo ese concepto (de ahí su elección en este artículo), y no sólo hablando de ese concepto de forma muy explícita en casi todos sus anuncios, sino también habiendo creado un Instituto de la felicidad ${ }^{38} \mathrm{e}$ incluso dos congresos de la felicidad ${ }^{39}$. Anuncios como el de la Fábrica de la felicidad ${ }^{40}$ o eslóganes como Estás aquí para ser feliz ${ }^{41} \mathrm{o}$ Repartiendo felicidad desde $1886^{42}$ son algunos de los muchos ejemplos que se pueden encontrar en esta marca. Otros ejemplos se pueden ver en Bavaria y su eslogan de $L a$ felicidad no dura siempre ${ }^{43}$ o en H\&S y su Por una cabeza más feliz ${ }^{44}$.

La segunda opción es también muy utilizada por la publicidad, quizá más frecuente que la primera en la publicidad actual, ya que las marcas prefieren transmitir el concepto de felicidad de forma implícita, a través de sonrisas y gestos de felicidad. En estos anuncios se observa cómo el elegir ese producto o servicio anunciado ofrece felicidad al consumidor, pero esto se transmite a través de sonrisas, de grandes sonrisas. Como ejemplos actuales, se pueden encontrar el anuncio de Vitaldent ${ }^{45}$, Fanta ${ }^{46}$, Campofrío ${ }^{47}$ o Mutua Madrileña ${ }^{48}$, entre otros muchos que se pueden ver en los distintos medios de comunicación actualmente ${ }^{49}$.

${ }^{36}$ Anuncios:Anuncios, http://www.anuncios.com/anunciantes/masanuncios/1042999007501/ coca-cola-manda-equipo-embajadores.1.html. Web visitada el 26/10/2009.

${ }^{37}$ EGUIZÁBAL, R (2009): Industrias de la conciencia: una historia social de la publicidad en España (1975-2009), Barcelona, Península, 310.

${ }^{38}$ Coca Cola: Instituto de la Felicidad de Coca-Cola, http://www.institutodelafelicidad.com/, Web visitada el 20/11/2012.

${ }^{39}$ Diario Crítico: Diario crítico, http://www.diariocritico.com/ocio-cultura/sociedad/cocacola/congreso-sobre-la-felicidad/409444. Web visitada el 15/11/2012.

${ }^{40}$ Youtube: Youtube, http://www.youtube.com/watch?v=rRoXp2cL6UA. Web visitada el 20/11/2012.

${ }^{41}$ Youtube: Youtube, http://www.youtube.com/watch?v=tzc3FFJDKU8. Web visitada el 20/11/2012.

${ }^{42}$ Youtube: Youtube, http://www.youtube.com/watch?v=diBN9Dy3REA. Web visitada el 20/11/2012.

${ }^{43}$ Youtube: Youtube, http://www.youtube.com/watch? $\mathrm{v}=\mathrm{a} 6 \mathrm{drY} Y \mathrm{Crq} 84$. Web visitada el 20/11/2012.

${ }^{44}$ Youtube: Youtube, http://www.youtube.com/watch?v=pJ9myJudELs. Web visitada el 20-11-2012.

${ }^{45}$ Youtube: Youtube, http://www.youtube.com/watch?v=-bGN5LnnSk4. Web visitada el 20/11/2012.

${ }^{46}$ Youtube: Youtube, http://www.youtube.com/watch?v=q8Y0-34DCOA. Web visitada el 20/11/2012.

${ }^{47}$ Youtube: Youtube, http://www.youtube.com/watch?v=ICws_wMsKXM. Web visitada el 20/11/2012.

${ }^{48}$ Youtube: Youtube, http://www.youtube.com/watch?v=KDyMA41ydVI\&feature=related. Web visitada el 20/11/2012.

${ }^{49}$ Los anuncios seleccionados en este artículo han sido elegidos por introducir el concepto de felicidad de forma muy explícita, lo que los convierte en muy ejemplificadotes para nuestro objeto de estudio, que no es otro que el estudio del concepto de la 'felicidad publicitaria'. 
Pero la felicidad también se transmite de forma implícita a través del éxito, tan deseado en la sociedad actual. ¿Cómo el éxito da lugar a la felicidad? Es sencillo: si se tiene éxito se ve a uno más feliz. Un concepto instaurado, como los otros, pero también equivocado, como explica Auger ${ }^{50}$, cuando dice que:

El éxito no aumenta mi valor ni un ápice. (...) Cuando consigo el éxito me siento, naturalmente, mejor de lo que me sentía antes. Pero eso no quiere decir que me haya hecho mejor persona. Eso es volver a confundir a la persona con sus actos, creer que soy bueno porque acierto y malo porque actuó de forma inadecuada.

Se pueden observar ejemplos en el anuncio de Nocilla ${ }^{51}$, de San Miguel ${ }^{52}$, Mahou ${ }^{53}$ o de Loterías ${ }^{54}$, donde se ve ese concepto de éxito del que se ha hablado, en el que se llama al consumidor a perseguir sus sueños, a luchar por el éxito y alcanzar así la felicidad ansiada por todas las personas.

Y como colofón a estos ejemplos, se debe resaltar uno que constituye una crítica a esto de lo que se ha venido hablando en este artículo: la felicidad publicitaria. Es un anuncio de galleta de Carrefour ${ }^{55}$. En él se hace una parodia de esa felicidad publicitaria actual de la que hablamos.

\section{Conclusiones}

Como conclusión a este artículo, se puede decir que el concepto de felicidad publicitaria, aparece cuando la publicidad llega al consumidor de forma masiva, con la Revolución Industrial, momento también en el que la publicidad deja de hablar de mercancías para empezar a hablar de sueños y valores. Con el apoyo de los medios de comunicación, especialmente la televisión y el color que ella ofrece, la publicidad comienza a crear ese concepto de felicidad de una forma sencilla: creando primero una insatisfacción (ya sea material o psicológica) en el consumidor, que se encuentra resuelta con el producto o servicio que ofrece el anuncio. Ahora bien, como son muchas las marcas que han seguido la misma estrategia, es necesario destacar y eso se consigue gritando más fuerte que los demás, llegando de forma más profunda al corazón del consumidor, creando una insatisfacción más grande.

Lo cierto es que buena parte de la publicidad actual ha elegido de forma muy acertada el concepto de felicidad y lo ha hecho porque es lo que cualquier persona ansía. La felicidad es el objetivo y necesidad de todos los seres humanos y, de ahí, que ésta sea el mejor reclamo publicitario actual. Prueba de ello es que en todas las teorías filosóficas, la felicidad gira como uno de los conceptos principales y, aunque hay diver-

${ }^{50}$ AUGER, L. (1987): Ayudarse a si mismo. Una psicoterapia mediante la razón, Sal Térrea, Cantabria, 60.

${ }^{51}$ Youtube: Youtube, http://www.youtube.com/watch?v=Lbi2fD2Bmnc. Web visitada el 20/11/2012.

${ }_{52}^{52}$ Youtube: Youtube, http://www.youtube.com/watch?v=tfMKSzW340Q. Web visitada el 20/11/2012.

${ }_{53}^{53}$ Youtube: Youtube, http://www.youtube.com/watch?v=5wWccG-3mXg. Web visitada el 20/11/2012.

${ }^{54}$ Youtube: Youtube, http://www.youtube.com/watch?v=QOqCsQ5cFzY. Web visitada el 20/11/2012.

${ }^{55}$ Youtube: Youtube, http://www.youtube.com/watch?v=BT6EuHPQi6I. Web visitada el 20/11/2012. 
genciasacerca de cómo conseguirla, en todas se ofrece un concepto de felicidad mucho más profundo del que se plasma en la publicidad, bastante más superficial. La felicidad es algo efímero, tan efímero como el consumir un producto. Cuando lo compras y lo consumes, esa felicidad se va.

En cualquier caso, ese concepto de felicidad, bien sea transmitido de forma explícita (introduciendo la palabra como tal en el texto o eslogan del anuncio) o implícita (a través de gestos que evocan la felicidad), se ha convertido en eje principal de la publicidad actual y, de ahí, la necesidad de aproximarnos a él. Esa felicidad publicitaria ha planteado una nueva forma de ser feliz, practicada por todos los ciudadanos y que supone la manera más sencilla de cumplir sus aspiraciones o, al menos, de estar más cerca de ellas. Eso es un logro y alivio para muchos. No se puede negar.

\section{Bibliografía}

ARISTÓTELES (2000): Metafísica, Madrid, Biblioteca Básica Gredos.

ARISTÓTELES (2003): Ética Eudemia, Buenos Aires, Losada.

AUGER, L. (1987): Ayudarse a si mismo. Una psicoterapia mediante la razón, Sal Térrea, Cantabria.

BASSAT, L. (1998): El libro rojo de la publicidad, Madrid, Espasa.

CORTINA, A. Y MARTÍNEZ, E. (2008): Ética, Madrid, Akal.

COSTA, J. (1992): Reinventar la publicidad. Reflexiones desde las Ciencias Sociales, Madrid, Fundesco.

CHECA GODOY, A. (2007): Historia de la publicidad, Galicia, Netbiblo.

EGUIZÁBAL, R. (1998): Historia de la publicidad. Madrid, Eresma y Celeste.

EGUIZÁBAL, R. (2009): Industrias de la conciencia: una historia social de la publicidad en España (1975-2009), Barcelona, Península.

HELLÍN, P. A. (2006): Publicidad y valores posmodernos, Madrid, Miranda Comunicación.

HUXLEY, A. (1995): Un mundo feliz, México, Editores Mexicanos Unidos.

JENOFONTE. (1967). Recuerdos de Sócrates, Madrid, Alianza Editorial.

KANT, I. (1967): Fundamentación de la metafísica de las costumbres, Madrid, Colección Austral.

MARÍAS, J. (1941): Historia de la filosofia, Madrid, Alianza Editorial.

MADRID CÁNOVAS, S. (2006): Semiótica del discurso publicitario, Murcia, Universidad de Murcia.

MILL, J.S. (2007): El utilitarismo, Madrid, Alianza Editorial.

MIÑANA, J. (2003): El mono cansado. Reflexiones sobre publicidad, Barcelona, Sine Qua Non.

ORTEGA, E. (2004): La comunicación publicitaria, Madrid, Pirámide.

POWELL, J. (1996): La felicidad es una tarea interior, Cantabria, Sal Terrae.

ROBERTS, K. (2005): Lovemarks, el futuro más allá de las marcas, Barcelona, Urano. ROBINSON- CHRISS, D. (2005): Ética para todos,. Barcelona, Paidós.

ROVIRA, A. Y MIRALLES, F. (2008): Ideas sobre la felicidad, Madrid, Punto de Lectura.

SAINT- EXUPÉRY, A. (1998): El principito, Madrid, Alianza Amecé. 
SAVATER, F. (1998): Ética para Amador, Barcelona, Ariel.

ZUBIRI, X. (2005): El hombre, lo real y lo irreal, Madrid, Alianza Editorial.

Anuncios: Anuncios, http://www.anuncios.com/anunciantes/masanuncios/1042999007501/coca-cola-manda-equipo-embajadores.1.html. Web visitada el 26/10/2009.

Coca-Cola: Instituto de la Felicidad de Coca-Cola, http://www.institutodelafelicidad. com/, Web visitada el 20/11/2012.

Diario Crítico: Diario Crítico, http://www.diariocritico.com/ocio-cultura/sociedad/coca-cola/congreso-sobre-la-felicidad/409444. Web visitada el 15/11/2012.

Youtube: Youtube, http://www.youtube.com/watch?v=rRoXp2cL6UA. Web visitada el 20/11/2012.

Youtube: Youtube, http://www.youtube.com/watch?v=tzc3FFJDKU8. Web visitada el 20/11/2012.

Youtube: Youtube, http://www.youtube.com/watch? $v=$ diBN9Dy3REA. Web visitada el 20-11-2012.

Youtube: Youtube, http://www.youtube.com/watch?v=a6drYlCrq84. Web visitada el 20/11/2012.

Youtube: Youtube, http://www.youtube.com/watch?v=pJ9myJudELs. Web visitada el 20/11/2012.

Youtube: Youtube, http://www.youtube.com/watch?v=-bGN5LnnSk4. Web visitada el 20/11/2012.

Youtube: Youtube, http://www.youtube.com/watch? $\mathrm{v}=\mathrm{q} 8 \mathrm{Y} 0-34 \mathrm{DCOA}$. Web visitada el 20/11/2012.

Youtube: Youtube, http://www.youtube.com/watch?v=ICws_wMsKXM. Web visitada el 20/11/2012.

Youtube: Youtube, http://www.youtube.com/watch?v=KDyMA41ydVI\&feature=relat ed. Web visitada el 20/11/2012.

Youtube: Youtube, http://www.youtube.com/watch?v=Lbi2fD2Bmnc. Web visitada el 20/11/2012.

Youtube: Youtube, http://www.youtube.com/watch?v=tfMKSzW340Q. Web visitada el 20/11/2012.

Youtube: Youtube, http://www.youtube.com/watch?v=5wWccG-3mXg. Web visitada el 20/11/2012.

Youtube: Youtube, http://www.youtube.com/watch?v=QOqCsQ5cFzY. Web visitada el 20/11/2012.

Youtube: Youtube, http://www.youtube.com/watch?v=BT6EuHPQi6I. Web visitada el 20/11/2012. 